\title{
INVARIANTE DIVISOREN UND SCHNITTHOMOLOGIE VON TORISCHEN VARIETÄTEN
}

\author{
GOTTFRIED BARTHEL \\ Fakultät für Mathematik, Universität Konstanz \\ Postfach 5560 D 203, D-78434 Konstanz, Germany \\ E-mail: Gottfried.Barthel@uni-konstanz.de \\ JEAN-PAUL BRASSELET \\ IML - CNRS: Singularités en Géométrie et Topologie Algébrique \\ IML Luminy Case 930, F-13288 Marseille Cedex 9, France \\ E-mail: jpb@iml.univ-mrs.fr \\ KARL-HEINZ FIESELER \\ Matematiska Institutionen, Uppsala Universitet \\ Box 480, S-751 06 Uppsala, Sweden \\ E-mail: khf@math.uu.se \\ LUDGER KAUP \\ Fakultät für Mathematik, Universität Konstanz \\ Postfach 5560 D 203, D-78434 Konstanz, Germany \\ E-mail: Ludger.Kaup@uni-konstanz.de
}

\begin{abstract}
In this article, we complete the interpretation of groups of classes of invariant divisors on a complex toric variety $X$ of dimension $n$ in terms of suitable (co-) homology groups. In $[\mathrm{BBFK}]$, we proved the following result (see Satz 1 below): Let $\mathrm{Cl} \operatorname{Div}_{\mathrm{C}}^{\mathbb{T}}(X)$ and $\mathrm{Cl} \operatorname{Div}_{\mathrm{W}}^{\mathbb{T}}(X)$ denote the groups of classes of invariant Cartier resp. Weil divisors on $X$. If $X$ is non degenerate (i.e., not equivariantly isomorphic to the product of a toric variety and a torus of positive dimension), then the natural homomorphisms $\mathrm{ClDiv}_{\mathrm{C}}^{\mathbb{T}}(X) \rightarrow H^{2}(X)$ and $\operatorname{ClDiv}_{\mathrm{W}}^{\mathbb{T}}(X) \rightarrow H_{2 n-2}^{\text {cld }}(X)$ are isomorphisms, the inclusion $\operatorname{ClDiv}_{\mathrm{C}}^{\mathbb{T}}(X) \hookrightarrow \mathrm{ClDiv}_{\mathrm{W}}^{\mathbb{T}}(X)$ corresponds to the Poincaré duality

1991 Mathematics Subject Classification: Primary 14M25, 14C20, 55N33; Secondary 52B20, $32 \mathrm{M} 12$.

Unterstützt durch das Programm „PROCOPE“ zur Förderung der deutsch-französischen Kooperation in der Forschung.

Avec le concours du programme «PROCOPE» pour l'avancement de la coopération francoallemande dans la recherche scientifique.

The paper is in final form and no version of it will be published elsewhere.
\end{abstract}


homomorphism $P_{2 n-2}$, and we have $H_{2 n-1}^{\text {cld }}(X) \cong H^{1}(X)=0$. For the convenience of the reader, the proof is sketched below; it supersedes the proof for the compact case given in the report $[\mathrm{BF}]$. Using suitable Künneth formulæ, that yields results valid in the degenerate case.

In the present article, we use the sheaf-theoretic description of the intersection homology groups $I_{\mathfrak{p}} H_{\bullet}^{\text {cld }}(X)$, for a perversity $\mathfrak{p}$, to prove that there is an open invariant subset $V_{\mathfrak{p}}$ of $X$ and a natural isomorphism $I_{\mathfrak{p}} H_{2 n-j}^{\text {cld }}(X) \cong H^{j}\left(V_{\mathfrak{p}}\right)$ for $j \leqq 2$. In the non degenerate case, we thus obtain an identification of $I_{\mathfrak{p}} H_{2 n-2}^{\text {cld }}(X)$ with $\mathrm{Cl}_{\operatorname{Div}} \mathbb{\mathfrak { p }}(X)$, the group of invariant Weil divisors on $X$ that are Cartier divisors on $V_{\mathfrak{p}}$, and the vanishing result $I_{\mathfrak{p}} H_{2 n-1}^{\text {cld }}(X)=0$ (see Satz 2). That divisor class group admits an explicit description in terms of the fan defining the toric variety. We use these results to treat problems of invariance of the intersection homology Betti number $I_{\mathfrak{p}} b_{2 n-2}^{\text {cld }}$. Moreover, we discuss the question when the homology Chern class $c_{n-1}(X)$ lies in the subgroup $I_{\mathfrak{p}} H_{2 n-2}^{\text {cld }}(X)$ of $H_{2 n-2}^{\text {cld }}(X)$.

\section{Zur Erinnerung an Krzysztof Jaczewski}

Einführung. Für eine glatte kompakte komplexe torische Varietät $X$ der (komplexen) Dimension $n$ lautet die „divisorielle“ Teilaussage des Satzes von Jurkiewicz und Danilov, daß ihre ganzzahlige Homologie $\mathrm{H}_{2 n-2}(X)$ mit der Divisorenklassengruppe und sogar mit der Gruppe $\mathrm{ClDiv}^{\mathbb{T}}(X)$ der Klassen von $\mathbb{T}$-invarianten Divisoren übereinstimmt, wobei $\mathbb{T}$ den auf $X$ operierenden eingebetteten Torus bezeichnet: Der kanonische „Homologieklassen-Homomorphismus" $\kappa: \mathrm{ClDiv}^{\mathbb{T}}(X) \rightarrow H_{2 n-2}(X)$ ist ein Isomorphismus. Per Poincaré-Dualität ergibt sich die analoge Aussage in der Kohomologie: Der „Chernklassen-Homomorphismus" $c^{1}: \mathrm{ClDiv}^{\mathbb{T}}(X) \rightarrow H^{2}(X)$ ist ein Isomorphismus.

Für eine singuläre torische Varietät $X$ ist zwischen Weil- und Cartierdivisoren zu unterscheiden, und neben der Kohomologie $H^{\bullet}(X)$ und der , üblichen“ Homologie $H_{\bullet}(X)$ mit kompakten Trägern sind die Schnitthomologie $I_{\mathfrak{p}} H_{\bullet}(X)$ zu einer beliebigen Toleranzfunktion $\mathfrak{p}$ ("perversity") sowie zusätzlich, wenn $X$ nicht notwendig kompakt ist, noch die Homologie $H_{\bullet}^{\text {cld }}(X)$ bzw. die Schnitthomologie $I_{\mathfrak{p}} H_{\bullet}^{\text {cld }}(X)$ mit abgeschlossenen Trägern zu betrachten. ${ }^{1)}$ Die natürlichen Homomorphismen $c^{1}: \operatorname{ClDiv}_{\mathrm{C}}^{\mathbb{T}}(X) \rightarrow H^{2}(X)$ sowie $\kappa: \operatorname{ClDiv}_{\mathrm{W}}^{\mathbb{T}}(X) \rightarrow H_{2 n-2}^{\text {cld }}(X)$ sind nun im allgemeinen keine Isomorphismen; als Hauptergebnis der Arbeit [BBFK] können wir aber den „divisoriellen“ Teil des Satzes von Jurkiewicz und Danilov über den kompakten Spezialfall (siehe [BF]) hinaus auf folgenden allgemeinen Fall übertragen: Wir nennen eine torische Varietät $X$ nicht entartet, wenn sich von $X$ kein nicht-trivialer Torusfaktor äquivariant abspalten läßt, d.h. wenn es keine echte Produktdarstellung $(X, \mathbb{T}) \cong\left(Y, \mathbb{T}^{\prime}\right) \times \mathbb{T}^{\prime \prime}$ gibt, wobei $\mathbb{T} \cong \mathbb{T}^{\prime} \times \mathbb{T}^{\prime \prime}$ eine Zerlegung des Torus und $Y$ eine torische Varietät bzgl. $\mathbb{T}^{\prime}$ ist. Es gilt dann:

SATz 1: Für eine nicht entartete komplexe torische Varietät $X$ mit $\operatorname{dim} X=n$ sind die natürlichen Homomorphismen $c^{1}: \operatorname{ClDiv}_{\mathrm{C}}^{\mathbb{T}}(X) \rightarrow H^{2}(X)$ sowie $\kappa: \operatorname{ClDiv}_{\mathrm{W}}^{\mathbb{T}}(X) \rightarrow$

Der „feder“-führende erstgenannte Autor (G.B.) dankt Piotr Pragacz für die Einladung zu der Tagung am Banach Center, für die Anregung, einen Beitrag zum Tagungsbericht einzureichen, und für die erwiesene Geduld bis zum Erhalt des Textes. Dem Max Planck -Institut für Mathematik in Bonn und dessen Leiter F. Hirzebruch dankt er für die finanzielle Unterstützung der Reise nach Warschau. Ein besonderer Dank, auch namens der beiden Konstanzer bzw. exKonstanzer Koautoren (K.-H.F. und L.K.), gilt unseren polnischen Kollegen und Freunden für die oft erwiesene herzliche Gastfreundschaft.

1) Die hier betrachteten (Schnitt-) Homologie- und Kohomologiegruppen haben stets ganzzahlige Koeffizienten. 
$H_{2 n-2}^{\text {cld }}(X)$ Isomorphismen, und das Diagramm

(D)

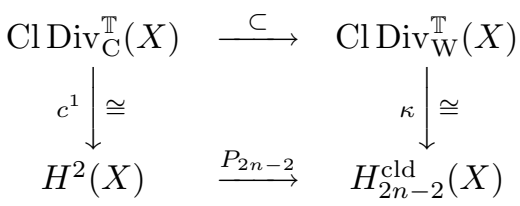

ist kommutativ. Damit ist der singuläre Poincaré-Dualitätshomomorphismus

$$
P_{2 n-2}: H^{2}(X) \rightarrow H_{2 n-2}^{\text {cld }}(X)
$$

stets injektiv. Weiter gilt

$$
H^{1}(X) \cong H_{2 n-1}^{\mathrm{cld}}(X)=0 .
$$

Im entarteten Fall folgen aus Satz 1 entsprechende Aussagen mit Hilfe von Künnethformeln: Man erhält Isomorphismen

$$
\mathrm{ClDiv}_{\mathrm{C}}^{\mathbb{T}}(X) \cong \operatorname{ClDiv}_{\mathrm{C}}^{\mathbb{T}}(Y) \text { sowie } \operatorname{ClDiv}_{\mathrm{W}}^{\mathbb{T}}(X) \cong \operatorname{ClDiv}_{\mathrm{W}}^{\mathbb{T}}(Y)
$$

und, falls $\mathbb{T}^{\prime \prime}$ maximal - also $Y$ nicht entartet - ist, ein kommutatives Diagramm

$$
\begin{array}{ccc}
\mathrm{ClDiv}_{\mathrm{C}}^{\mathbb{T}}(X) \oplus H^{2}\left(\mathbb{T}^{\prime \prime}\right) & \longrightarrow & \operatorname{ClDiv}_{\mathrm{W}}^{\mathbb{T}}(X) \oplus H_{2 n-2 d-2}^{\text {cld }}\left(\mathbb{T}^{\prime \prime}\right) \\
c^{1} \oplus \operatorname{pr}^{*} \downarrow & \cong & \kappa \oplus \mathrm{pr}^{*} \downarrow \cong \\
H^{2}(X) & \stackrel{P_{2 n-2}(X)}{\longrightarrow} & H_{2 n-2}^{\text {cld }}(X),
\end{array}
$$

wobei der obere Pfeil die direkte Summe der Inklusion $\operatorname{ClDiv}_{\mathrm{C}}^{\mathbb{T}}(X) \hookrightarrow \operatorname{ClDiv}_{\mathrm{W}}^{\mathbb{T}}(X)$ und des Poincaré-Homomorphismus $P_{2 n-2 d-2}\left(\mathbb{T}^{\prime \prime}\right)$ ist; weiter gilt dann

$$
H^{1}(X) \cong H_{2 n-1}^{\text {cld }}(X) \cong H^{1}\left(\mathbb{T}^{\prime \prime}\right) \text {. }
$$

In dieser Note zeigen wir, daß Satz 1 ein Spezialfall eines allgemeineren Resultates über die Schnitthomologie $I_{\mathfrak{p}} H_{\bullet}^{\text {cld }}(X)$ für beliebige Toleranz $\mathfrak{p}$ ist. Dazu sei $V_{\mathfrak{p}}$ die offene invariante Teilmenge von $X$, die aus allen Torusbahnen $B$ mit $\operatorname{codim}_{X}^{\mathbb{C}} B \leqq i(\mathfrak{p}):=$ $\max \{i ; i \leqq n, \mathfrak{p}(2 i) \leqq 1\}$ besteht. Weiter bezeichne

$$
\operatorname{ClDiv}_{\mathfrak{p}}^{\mathbb{T}}(X):=\left\{[D] \in \operatorname{ClDiv}_{W}^{\mathbb{T}}(X) ;\left.D\right|_{V_{\mathfrak{p}}} \in \operatorname{Div}_{\mathrm{C}}^{\mathbb{T}}\left(V_{\mathfrak{p}}\right)\right\}
$$

die Gruppe der Klassen solcher invarianten Weildivisoren auf $X$, deren Einschränkung auf $V_{\mathfrak{p}}$ ein Cartierdivisor ist; diese ist offensichtlich kanonisch zur Gruppe $\mathrm{Cl} \operatorname{Div}_{\mathrm{C}}^{\mathbb{T}}\left(V_{\mathfrak{p}}\right)$ isomorph. Mit diesen Bezeichnungen gilt

SATz 2: Für eine nicht entartete komplexe torische Varietät $X$ mit $\operatorname{dim} X=n$ gibt es natürliche Isomorphismen $\mathrm{ClDiv}_{\mathfrak{p}}^{\mathbb{T}}(X) \stackrel{\cong}{\rightrightarrows} I_{\mathfrak{p}} H_{2 n-2}^{\text {cld }}(X)$, die das Diagramm (D) aus Satz 1 wie folgt kommutativ ergänzen:

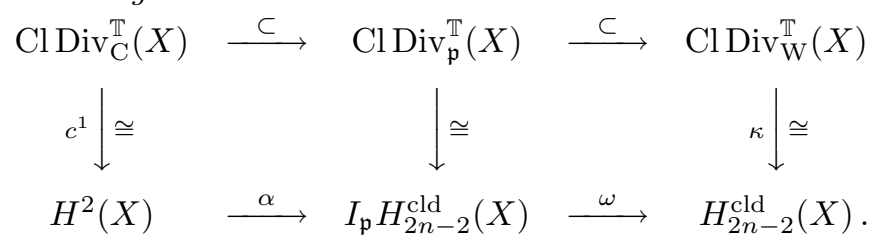

Dabei ist die untere Zeile die kanonische Faktorisierung des Poincaré-Homomorphismus $P_{2 n-2}$ durch die Vergleichshomomorphismen

$$
\alpha=\alpha_{\mathfrak{p}}: H^{2}(X) \rightarrow I_{\mathfrak{p}} H_{2 n-2}^{\text {cld }}(X) \quad \text { und } \quad \omega=\omega_{\mathfrak{p}}: I_{\mathfrak{p}} H_{2 n-2}^{\text {cld }}(X) \rightarrow H_{2 n-2}^{\text {cld }}(X) ;
$$


insbesondere sind diese Homomorphismen stets injektiv. Weiter gilt

$$
I_{\mathfrak{p}} H_{2 n-1}^{\text {cld }}(X)=0 .
$$

Somit können wir alle Schnitthomologiegruppen $I_{\mathfrak{p}} H_{2 n-2}^{\text {cld }}(X)$ - auch die besonders interessante „selbst-duale“ Schnitthomologie

$$
I H_{2 n-2}^{\mathrm{cld}}(X):=I_{\mathfrak{m}} H_{2 n-2}^{\mathrm{cld}}(X)
$$

zur mittleren Toleranz $\mathfrak{m}$ mit $i(\mathfrak{m})=2$ - als Untergruppen von $H_{2 n-2}^{\text {cld }}(X)$ auffassen. Für die beiden ,extremen“ Toleranzfunktionen $\mathfrak{p}=\mathfrak{o}$ bzw. $\mathfrak{p}=\mathfrak{t}$ gibt es bekanntlich natürliche Isomorphismen

$$
I_{\mathrm{o}} H_{2 n-\bullet}^{\text {cld }}(X) \cong H^{\bullet}(X) \quad \text { bzw. } \quad I_{\mathrm{t}} H_{2 n-\bullet}^{\text {cld }} .(X) \cong H_{2 n-\bullet}^{\text {cld }}(X) ;
$$

weiter gilt $i(\mathfrak{o})=n$ bzw. $i(\mathfrak{t})=1$, also $V_{\mathfrak{o}}=X$ sowie $V_{\mathfrak{t}} \subset X_{\text {reg }}$ und deshalb $\operatorname{Div}_{\mathfrak{o}}^{\mathbb{T}}(X) \cong$ $\operatorname{Div}_{\mathrm{C}}^{\mathbb{T}}(X)$ sowie $\operatorname{Div}_{\mathfrak{t}}^{\mathbb{T}}(X) \cong \operatorname{Div}_{\mathrm{W}}^{\mathbb{T}}(X)$, womit sich wieder die Aussagen von Satz 1 ergeben.

Im entarteten Fall erhalten wir ähnlich wie beim Zusatz zu Satz 1 natürliche Isomorphismen $I_{\mathfrak{p}} H_{2 n-2}^{\text {cld }}(X) \cong \mathrm{ClDiv}_{\mathfrak{p}}^{\mathbb{T}}(X) \oplus H^{2}\left(\mathbb{T}^{\prime \prime}\right)$, die das Diagramm $\left(\mathrm{D}^{\prime}\right)$ kommutativ ergänzen.

Als Anwendung diskutieren wir, wie die Schnitt-Bettizahl $I_{\mathfrak{p}} b_{2 n-2}^{\text {cld }}(X)$ vom definierenden Fächer abhängt und wann die Homologie-Chernklasse $c_{n-1}(X)$ schon in der Untergruppe $I_{\mathfrak{p}} H_{2 n-2}^{\text {cld }}(X)$ von $H_{2 n-2}^{\text {cld }}(X)$ liegt.

Für die Theorie torischer Varietäten verweisen wir auf die Monographien [Fu] und [Od], zur Schnitthomologie auf [Bo], und auf die jeweils erwähnte Literatur.

Wir widmen diesen Artikel dem Andenken an unseren Warschauer Freund und Kollegen Krzysztof Jaczewski. Durch sein offenes und herzliches Wesen, seinen Enthusiasmus und sein Wissen bereicherte er jedes Zusammentreffen auf den jährlichen HerbstSeminaren der Arbeitsgruppe um Andrzej Białynicki-Birula, an denen wir im Rahmen der Kooperation zwischen den Universitäten Warschau und Konstanz teilnehmen konnten. Die Begegnung beim Workshop "Parameter Spaces" am Banach Center im Februar 1994 blieb die letzte. Sein plötzlicher Tod im September 1994, nur wenige Tage vor dem schon erwarteten nächsten Treffen beim Herbst-Seminar in Wikno, wo die hier publizierten Ergebnisse erstmals präsentiert wurden, hat uns tief berührt. Er wird uns fehlen.

0. Grundlagen und Bezeichnungen: Es sei $\mathbb{T} \cong\left(\mathbb{C}^{*}\right)^{n}$ ein $n$-dimensionaler komplex-algebraischer Torus, $N:=\operatorname{Hom}\left(\mathbb{C}^{*}, \mathbb{T}\right) \cong \mathbb{Z}^{n}$ die Gruppe der Ein-Parameter-Untergruppen und $M:=\operatorname{Hom}\left(\mathbb{T}, \mathbb{C}^{*}\right)$ die Charaktergruppe des Torus. Weiter bezeichne $\left\langle_{-},{ }_{-}\right\rangle$: $M \times N \rightarrow \mathbb{Z}$ die duale Paarung, die durch Komposition und die kanonische Identifikation $\operatorname{Hom}\left(\mathbb{C}^{*}, \mathbb{C}^{*}\right) \cong \mathbb{Z}$, also durch $\chi(v(\lambda))=\lambda^{\langle\chi, v\rangle}$ für $\chi \in M, v \in N$ und $\lambda \in \mathbb{C}^{*}$, gegeben ist. Damit können wir $M$ und $N$ als Paar zueinander dualer Gitter in den zueinander dualen reellen Vektorräumen $M_{\mathbb{R}}:=M \otimes_{\mathbb{Z}} \mathbb{R} \cong \mathbb{R}^{n}$ und $N_{\mathbb{R}}:=N \otimes_{\mathbb{Z}} \mathbb{R} \cong \mathbb{R}^{n}$ auffassen.

Eine torische Varietät $X$ mit eingebettetem Torus $\mathbb{T}$ ist eine normale komplex-algebraische Varietät mit einer effektiven und fast transitiven Operation des Torus $\mathbb{T}$, d.h. es gibt eine offene dichte Bahn $B_{o}$, so daß die Bahnabbildung $\mathbb{T} \rightarrow B_{o}, t \mapsto t x_{0}$ zu einem Basispunkt $x_{0} \in B_{o}$ ein Isomorphismus ist. Einer solchen Varietät entspricht umkehrbar eindeutig ein ( $N$-rationaler endlicher) Fächer $\Delta$ in $N_{\mathbb{R}}$, d.h. eine endliche Familie von $\left(N\right.$-rationalen strikt konvexen polyedrischen) Kegeln in $N_{\mathbb{R}}$, so daß mit jedem Kegel $\sigma$ auch jede Seite $\tau \prec \sigma$ zu $\Delta$ gehört und sich je zwei Kegel in einer gemeinsamen Seite 
schneiden. Ein derartiger Kegel $\sigma$ ist definiert als Menge

$$
\operatorname{pos} \operatorname{lin} P(\sigma):=\left\{\sum_{v \in P(\sigma)} \lambda_{v} \cdot v ; \lambda_{v} \in \mathbb{R}_{\geqq 0}\right\} \subset N_{\mathbb{R}}
$$

der positiven Linearkombinationen einer endlichen Menge $P(\sigma) \subset N$ von primitiven Gittervektoren, die in einem Halbraum $\{v ;\langle\chi, v\rangle>0\}$ von $N_{\mathbb{R}}$ (mit geeignetem $\chi \in M$ ) enthalten ist. Dieses primitive Erzeugendensystem $P(\sigma)$ sei zusätzlich minimal gewählt, d.h. alle Strahlen $\rho_{v}:=\mathbb{R}_{\geqq 0} \cdot v$ für $v \in P(\sigma)$ sind auch Seiten des Kegels; wir setzen $P(\Delta):=\bigcup_{\sigma \in \Delta} P(\sigma)$. Jeder solche Kegel $\sigma$ bestimmt eine affine torische Varietät $X_{\sigma}$, und für jede Seite $\tau \prec \sigma$ gibt es eine kanonische offene äquivariante Einbettung $X_{\tau} \stackrel{\varsigma}{\rightarrow}$ $X_{\sigma}$. Durch äquivariantes Verkleben ergibt sich dann eine torische Varietät $X_{\Delta}$ mit einer invarianten affinen offenen Überdeckung $\left(X_{\sigma}\right)_{\sigma \in \Delta}$.

Offenbar ist ein Fächer $\Delta$ schon durch die Menge $\Delta^{\max }$ seiner maximalen Kegel erzeugt, und die zugehörigen invarianten affinen offenen Mengen $X_{\sigma}$ überdecken $X_{\Delta}$; im Spezialfall $\Delta^{\max }=\{\sigma\}$ ist $X_{\Delta}$ die affine torische Varietät $X_{\sigma}$. Die Varietät $X_{\Delta}$ ist genau dann kompakt, wenn der Fächer $\Delta$ vollständig ist, d.h. wenn $\bigcup_{\sigma \in \Delta} \sigma=N_{\mathbb{R}}$ gilt, und $X_{\Delta}$ ist genau dann nicht entartet, wenn $\Delta$ nicht entartet, d.h. in keiner Hyperebene von $N_{\mathbb{R}}$ enthalten, ist. Eine affine torische Varietät $X_{\sigma}$ ist genau dann glatt, wenn der Kegel $\sigma$ regulär ist, d.h. wenn $P(\sigma)$ zu einer Gitterbasis von $N$ ergänzt werden kann; es gilt dann

$$
X_{\sigma} \cong \mathbb{C}^{d} \times\left(\mathbb{C}^{*}\right)^{n-d} \text { mit } \quad d:=d(\sigma):=\operatorname{dim} \sigma .
$$

Allgemeiner heißt ein Kegel $\sigma$ simplizial, wenn $P(\sigma)$ ein linear unabhängiges System ist. In diesem Fall ist $X_{\sigma}$ eine rationale Homologiemannigfaltigkeit, denn es gilt

$$
X_{\sigma} \cong\left(\mathbb{C}^{d} / \Gamma_{\sigma}\right) \times\left(\mathbb{C}^{*}\right)^{n-d},
$$

wobei $\Gamma_{\sigma} \subset\left(\mathbb{C}^{*}\right)^{d} \subset \mathrm{GL}_{d}(\mathbb{C})$ eine zu $(N \cap \operatorname{lin} \sigma) /\langle P(\sigma)\rangle$ isomorphe endliche Gruppe von Diagonalmatrizen ist. - Der Fächer $\Delta$ heißt regulär bzw. simplizial, wenn alle seine Kegel regulär bzw. simplizial sind. Insbesondere ist

$$
\Delta^{(\leqq d)}:=\bigcup_{j \leqq d} \Delta^{(j)} \quad\left(\operatorname{mit} \quad \Delta^{(j)}:=\{\sigma \in \Delta ; \operatorname{dim} \sigma=j\}\right)
$$

für $d=1$ ein regulärer und für $d=2$ ein simplizialer Fächer.

Einem Kegel $\sigma$ entspricht eine $(n-d)$-dimensionale Bahn $B_{\sigma}$ des Torus $\mathbb{T}$, die in der affinen torischen Varietät $X_{\sigma}$ liegt und dort die einzige abgeschlossene Bahn ist. Ist $d=n$, so erhalten wir eine einpunktige Bahn $B_{\sigma}=:\left\{x_{\sigma}\right\}$, also einen Fixpunkt, und $X_{\sigma}$ ist äquivariant auf $x_{\sigma}$ zusammenziehbar. Ist $d<n$, so gibt es einen äquivarianten Isomorphismus $\left(X_{\sigma}, B_{\sigma}\right) \cong\left(Y_{\sigma},\left\{y_{\sigma}\right\}\right) \times\left(\mathbb{C}^{*}\right)^{n-d}$, wobei $Y_{\sigma}$ die auf ihren Fixpunkt $y_{\sigma}$ zusammenziehbar affine torische Varietät ist, die von $\sigma$ bezüglich des Untervektorraums $\operatorname{lin} \sigma$ und des Gitters $N \cap \operatorname{lin} \sigma$ definiert wird. - Die $(n-1)$-dimensionalen Bahnen entsprechen umkehrbar eindeutig den Strahlen $\rho_{v}:=\mathbb{R}_{\geqq 0} v$ für $v \in P(\Delta)$; wir schreiben kurz $B_{v}$ statt $B_{\rho_{v}}$. - Für die durch $\Delta^{(\leqq d)}$ definierte offene invariante Teilmenge $V_{d}$ von $X_{\Delta}$ gilt $V_{d}=\bigcup_{d(\sigma) \leqq d} B_{\sigma}$.

Als Folgerung aus der Formel $\pi_{1}\left(X_{\Delta}\right) \cong N /\left(\sum_{\sigma \in \Delta} N \cap \operatorname{lin} \sigma\right)$ für die Fundamentalgruppe einer torischen Varietät bemerken wir, daß $\Delta$ genau dann nicht entartet ist, wenn die abelsche Gruppe $\pi_{1}\left(X_{\Delta}\right)=H_{1}\left(X_{\Delta}\right)$ endlich ist; dazu ist auch äquivalent, daß $H^{1}\left(X_{\Delta}\right)$ verschwindet. 
1. Invariante Divisoren. Die Gruppen der Weil-, Cartier- bzw. Haupt-Divisoren und die zugehörigen Divisorenklassengruppen bezeichnen wir mit $\operatorname{Div}_{\mathrm{W}}(X), \operatorname{Div}_{\mathrm{C}}(X)$, $\operatorname{Div}_{0}(X)$ sowie $\mathrm{Cl} \operatorname{Div}_{\mathrm{W}}(X)$ bzw. $\mathrm{ClDiv}_{\mathrm{C}}(X)$. Wir schreiben

$$
\operatorname{Div}_{\mathrm{W}}^{\mathbb{T}}(X) \supset \operatorname{Div}_{\mathrm{C}}^{\mathbb{T}}(X) \supset \operatorname{Div}_{0}^{\mathbb{T}}(X)
$$

sowie $\mathrm{ClDiv}_{\mathrm{W}}^{\mathbb{T}}(X)$ bzw. $\mathrm{ClDiv} \mathrm{C}_{\mathrm{C}}^{\mathbb{T}}(X)$ für die entsprechend mit $\mathbb{T}$-invarianten Divisoren gebildeten Gruppen. Ein Divisor auf $X$ ist genau dann invariant, wenn sein Träger disjunkt zum eingebetteten Torus $B_{o} \cong \mathbb{T}$ ist. Die folgende einfache Bemerkung zeigt die besondere Bedeutung der invarianten Divisoren:

B e merkung: Jeder Divisor auf der komplexen torischen Varietät $X$ ist linear äquivalent zu einem $\mathbb{T}$-invarianten Divisor; es gibt also Isomorphismen

$$
\mathrm{ClDiv} \mathbb{C}_{\mathrm{T}}^{\mathbb{T}}(X) \cong \operatorname{ClDiv}_{\mathrm{C}}(X) \text { und } \operatorname{ClDiv}_{\mathrm{W}}^{\mathbb{T}}(X) \cong \operatorname{ClDiv}_{\mathrm{W}}(X) \text {. }
$$

Der B ew eis ergibt sich sofort daraus, daß der Koordinatenring

$$
\mathcal{O}(\mathbb{T}) \cong \mathbb{C}\left[z_{1}, z_{1}^{-1}, \ldots, z_{n}, z_{n}^{-1}\right]
$$

des Torus faktoriell ist und somit ein beliebiger Divisor auf dem eingebetteten Torus stets mit einem Hauptdivisor übereinstimmt.

Die invarianten Primdivisoren sind genau die Abschlüsse $\bar{B}_{v}$ der $(n-1)$-dimensionalen Bahnen; somit entsprechen sie den „Basisvektoren“ $v \in P(\Delta)$ der Strahlen, und wir erhalten einen natürlichen Isomorphismus

$$
\operatorname{Div}_{\mathrm{W}}^{\mathbb{T}}(X) \cong \bigoplus_{v \in P(\Delta)} \mathbb{Z} \cdot \bar{B}_{v}
$$

Die Gruppe $\operatorname{Div}_{0}^{\mathbb{T}}(X)$ der invarianten Hauptdivisoren ist das Bild der Charaktergruppe $M$ unter dem natürlichen Homomorphismus $\operatorname{div}_{0}: M \rightarrow \operatorname{Div}_{\mathrm{W}}^{\mathbb{T}}(X), \chi \mapsto \operatorname{div}_{0}(\chi):=$ $\sum_{v \in P(\Delta)}\langle\chi, v\rangle \cdot \bar{B}_{v}$, weil jede invertierbare reguläre Funktion auf dem Torus ein LaurentMonom und somit Vielfaches eines Charakters ist. Für einen nicht entarteten Fächer $\Delta$ liefert $\operatorname{div}_{0}$ einen Isomorphismus $M \cong \operatorname{Div}_{0}^{\mathbb{T}}(X)$.

Zur expliziten Beschreibung der Untergruppe $\operatorname{Div}_{\mathrm{C}}^{\mathbb{T}}(X)$ (siehe [BBFK: $\left.\S 1 . \mathrm{D}\right]$ ) benutzen wir, daß invariante Cartier-Divisoren auf einer affinen offenen Menge $X_{\sigma}$ stets Hauptdivisoren sind, also $\operatorname{Div}_{\mathrm{C}}^{\mathbb{T}}\left(X_{\sigma}\right)=\operatorname{Div}_{0}^{\mathbb{T}}\left(X_{\sigma}\right)$ und somit

$$
\operatorname{Div}_{\mathrm{C}}^{\mathbb{T}}\left(X_{\Delta}\right)=\bigcap_{\sigma \in \Delta_{\text {max }}} G_{\sigma} \quad \text { mit } \quad G_{\sigma}:=\left\{D \in \operatorname{Div}_{\mathrm{W}}^{\mathbb{T}}\left(X_{\Delta}\right) ;\left.D\right|_{X_{\sigma}} \in \operatorname{Div}_{0}^{\mathbb{T}}\left(X_{\sigma}\right)\right\}
$$

gilt. Demnach liegt ein invarianter Divisor $D=\sum_{v \in P(\Delta)} a_{v} \cdot \bar{B}_{v} \in \operatorname{Div}_{\mathrm{W}}^{\mathbb{T}}(X)$ genau dann in $\operatorname{Div}_{C}^{\mathbb{T}}(X)$, wenn zu jedem maximalen Kegel $\sigma$ ein Charakter $\chi_{\sigma}$ mit $\left.D\right|_{X_{\sigma}}=\operatorname{div}\left(\chi_{\sigma}\right)$, d.h. $a_{v}=\left\langle\chi_{\sigma}, v\right\rangle$ für jedes $v \in P(\sigma)$, existiert. Dieser Charakter $\chi_{\sigma} \in M$ ist nur modulo der Untergruppe $P(\sigma)^{\perp}:=M \cap \sigma^{\perp}$ eindeutig bestimmt; mit

$$
M^{[\Delta]}:=\left\{\left(\bar{\chi}_{\sigma}\right) \in \bigoplus_{\sigma \in \Delta^{\max }}\left(M / P(\sigma)^{\perp}\right) ; \forall_{\sigma, \sigma^{\prime} \in \Delta^{\max }} \forall_{v \in P(\sigma) \cap P\left(\sigma^{\prime}\right)}\left\langle\chi_{\sigma}, v\right\rangle=\left\langle\chi_{\sigma^{\prime}}, v\right\rangle\right\}
$$

erhalten wir nun einen Isomorphismus div : $M^{[\Delta]} \stackrel{\cong}{\rightrightarrows} \operatorname{Div}_{\mathrm{C}}^{\mathbb{T}}(X)$, indem wir einer Familie $\left(\bar{\chi}_{\sigma}\right)_{\sigma \in \Delta^{\max }}$ den Divisor $\sum_{v \in P(\Delta)}\left\langle\chi_{\sigma(v)}, v\right\rangle \cdot \bar{B}_{v}$ zuordnen, wobei jeweils zu $v$ ein Kegel $\sigma(v) \in \Delta^{\max }$ mit $v \in \sigma(v)$ zu wählen ist. Das folgende kommutative Diagramm beschreibt 
damit die verschiedenen Gruppen von invarianten Divisoren:

$$
\begin{array}{ccccc}
M & \stackrel{\operatorname{div}_{0}}{\longrightarrow} & \operatorname{div}_{0}(M) & \stackrel{D^{2}}{\operatorname{Div}_{0}^{\mathbb{T}}(X)} \\
\downarrow & & \cap & \cap \\
M^{[\Delta]} & \stackrel{\operatorname{div}}{\longrightarrow} & \operatorname{div}\left(M^{[\Delta]}\right) & = & \operatorname{Div}_{\mathrm{C}}^{\mathbb{T}}(X) \\
& & \cap & \cap & \cap \\
& & \bigoplus_{v \in P(\Delta)} \mathbb{Z} \cdot \bar{B}_{v} & \stackrel{\cong}{\longrightarrow} \operatorname{Div}_{\mathrm{W}}^{\mathbb{T}}(X) .
\end{array}
$$

Dabei bezeichnet der senkrechte Pfeil $M \rightarrow M^{[\Delta]}$ die „Diagonalabbildung“ $\chi \mapsto(\bar{\chi}) \in$ $M^{[\Delta]}$ auf die ,konstante“ Familie $(\bar{\chi})=\left(\bar{\chi}_{\sigma}\right)$ mit $\chi_{\sigma} \equiv \chi$.

Es gilt $\operatorname{rg} \operatorname{Div}_{\mathrm{W}}^{\mathbb{T}}\left(X_{\Delta}\right)=\operatorname{card} P(\Delta)=\operatorname{card} \Delta^{(1)}$ (und damit $\operatorname{rg} \operatorname{ClDiv}_{\mathrm{W}}^{\mathbb{T}}\left(X_{\Delta}\right)=$ card $\Delta^{(1)}-n$, falls $\Delta$ nicht entartet ist). Um die Differenz zum Rang der Untergruppe $\operatorname{Div}_{\mathrm{C}}^{\mathbb{T}}\left(X_{\Delta}\right)=\bigcap_{\sigma \in \Delta_{\max }} G_{\sigma}$ zu bestimmen, gehen wir zur orthogonalen Untergruppe $\operatorname{Div}_{\mathrm{C}}^{\mathbb{T}}\left(X_{\Delta}\right)^{\perp}$ in der dualen Gruppe $\operatorname{Hom}\left(\operatorname{Div}_{\mathrm{W}}^{\mathbb{T}}\left(X_{\Delta}\right), \mathbb{Z}\right)$ über: Diese enthält $\sum_{\sigma \in \Delta_{\max }} G_{\sigma}^{\perp}$ als Untergruppe von endlichem Index, und somit gilt

$$
\operatorname{rg} \operatorname{Div}_{W}^{\mathbb{T}}\left(X_{\Delta}\right)-\operatorname{rg} \operatorname{Div}_{\mathrm{C}}^{\mathbb{T}}\left(X_{\Delta}\right)=\operatorname{rg}\left(\sum_{\sigma \in \Delta^{\max }} G_{\sigma}^{\perp}\right)
$$

Mit den Identifikationen

$$
\operatorname{Div}_{\mathrm{W}}^{\mathbb{T}}\left(X_{\Delta}\right) \cong \mathbb{Z}^{P(\Delta)} \text { und } \operatorname{Hom}\left(\operatorname{Div}_{\mathrm{W}}^{\mathbb{T}}\left(X_{\Delta}\right), \mathbb{Z}\right) \cong \operatorname{Hom}\left(\mathbb{Z}^{P(\Delta)}, \mathbb{Z}\right)
$$

ergibt sich $G_{\sigma}=\left\{\left(a_{v}\right) \in \mathbb{Z}^{P(\Delta)} ; \exists_{\chi_{\sigma} \in M} \forall_{v \in P(\sigma)} a_{v}=\left\langle\chi_{\sigma}, v\right\rangle\right\}$ und

$$
G_{\sigma}^{\perp}=\left\{l \in \operatorname{Hom}\left(\mathbb{Z}^{P(\Delta)}, \mathbb{Z}\right) ; \sum_{v \in P(\sigma)} l\left(\varepsilon_{v}\right) \cdot v=0, \forall_{v \in P(\Delta) \backslash P(\sigma)} l\left(\varepsilon_{v}\right)=0\right\} .
$$

Weil $G_{\sigma}^{\perp}$ somit die linearen Relationen zwischen den Elementen des Erzeugendensystems $P(\sigma)$ im Gitter $N$ beschreibt, gilt $\operatorname{rg}\left(\sum_{\sigma \in \Delta^{\max }} G_{\sigma}^{\perp}\right)=\operatorname{rg} R$, wobei $R$ eine Relationenmatrix ist, die man wie folgt erhält: Für jeden Kegel $\sigma \in \Delta^{\max }$ fixiert man eine maximale linear unabhängige Teilmenge $P^{\prime}(\sigma) \subset P(\sigma)$. Da jedes Element $v \in P(\sigma) \backslash P^{\prime}(\sigma)$ von $P^{\prime}(\sigma)$ rational linear abhängig ist, gibt es genau eine ganzzahlige primitive lineare Relation $\sum_{w \in P(\Delta)} r_{v w}^{\sigma} \cdot w=0$ mit $r_{v w}^{\sigma}=0$ für $w \notin P^{\prime}(\sigma) \cup\{v\}$ und $r_{v v}^{\sigma}>0$. Die Koeffizientenfolgen $\left(r_{v w}^{\sigma}\right)_{w \in P(\Delta)}$ bilden die Zeilen der Matrix $R$, wobei $\sigma$ in $\Delta^{\text {max }}$ und zusätzlich $v$ in $P(\sigma) \backslash P^{\prime}(\sigma)$ variiert.

Offensichtlich ist ein Kegel $\sigma$ genau dann simplizial, wenn $G_{\sigma}^{\perp}=0$ gilt; ein solcher Kegel liefert keine Zeilen zu der Relationenmatrix. Somit ist der gesamte Fächer $\Delta$ genau dann simplizial, wenn die Matrix $R$ keine Zeile hat, also wenn $\operatorname{rg} R=0$ gilt. In diesem Fall ergibt sich $\operatorname{rg} \operatorname{Div}_{\mathrm{C}}^{\mathbb{T}}\left(X_{\Delta}\right)=\operatorname{rg} \operatorname{Div}_{\mathrm{W}}^{\mathbb{T}}\left(X_{\Delta}\right)=\operatorname{card} \Delta^{(1)}$.

2. Zum Beweis von Satz 1. Vor dem Beweis von Satz 2, der ganz entscheidend auf der Kohomologieaussage von Satz 1 beruht, skizzieren wir einige wesentliche Schritte zu deren Beweis.

Wir haben bereits gesehen, daß $X_{\Delta}$ genau dann nicht entartet ist, wenn $H^{1}\left(X_{\Delta}\right)$ verschwindet. Für die restlichen Behauptungen reduzieren wir zunächst auf den „minimalen“ Fall: Da der Fächer $\Delta$ nicht entartet ist, gibt es $n$ linear unabhängige Vektoren $v_{1}, \ldots, v_{n}$ in $P(\Delta)$. Die zugehörigen Strahlen $\mathbb{R}_{\geq 0} v_{j}$ bilden zusammen mit dem Nullkegel $o:=\{0\}$ einen minimalen nicht entarteten Teilfächer $\Delta^{\prime} \subset \Delta$, dem eine glatte offene invariante Teilmenge $X_{\Delta^{\prime}}$ von $X_{\Delta}$ entspricht. Diese ist gleichzeitig auch als offene invariante Teilmenge in der affinen torischen Varietät enthalten, die zu dem von $\left\{v_{1}, \ldots, v_{n}\right\}=P\left(\Delta^{\prime}\right)$ 
aufgespannten $n$-dimensionalen simplizialen Kegel gehört, und deshalb gibt es eine Darstellung $X_{\Delta^{\prime}} \cong Z / \Gamma$ als Quotient von $Z:=\mathbb{C}^{n} \backslash \bigcup_{1 \leqq i<j \leqq n}\left(z_{i}=z_{j}=0\right)$ nach der freien Operation einer endlichen Gruppe $\Gamma \subset\left(\mathbb{C}^{*}\right)^{n} \subset \mathrm{GL}_{n}(\mathbb{C})$ von Diagonalmatrizen, die zur Restklassengruppe $N / N^{\prime}$ mit $N^{\prime}:=\left\langle v_{1}, \ldots, v_{n}\right\rangle$ isomorph ist. Für die Mannigfaltigkeit $X_{\Delta^{\prime}}$ genügt es nun, die Homologieaussagen des Satzes zu beweisen: Da $Z$ einfach zusammenhängend ist, erhalten wir mit universellen Koeffizientensätzen und Poincaré-Dualität die Isomorphismen

$$
\Gamma \cong \pi_{1}\left(X_{\Delta^{\prime}}\right) \cong H_{1}\left(X_{\Delta^{\prime}}\right) \cong H^{2}\left(X_{\Delta^{\prime}}\right) \cong H_{2 n-2}^{\text {cld }}\left(X_{\Delta^{\prime}}\right)
$$

und $H_{2 n-1}^{\text {cld }}\left(X_{\Delta^{\prime}}\right) \cong H^{1}\left(X_{\Delta^{\prime}}\right)=0$. Weil also $H_{2 n-2}^{\text {cld }}\left(X_{\Delta^{\prime}}\right)$ eine Torsionsgruppe ist, folgt die Surjektivität von $\kappa: \mathrm{ClDiv}^{\mathbb{T}}\left(X_{\Delta^{\prime}}\right) \rightarrow H_{2 n-2}^{\text {cld }}\left(X_{\Delta^{\prime}}\right)$ mit Hilfe der natürlichen Identifikation $\operatorname{Div}^{\mathbb{T}}\left(X_{\Delta^{\prime}}\right) \cong H_{2 n-2}^{\text {cld }}\left(X_{\Delta^{\prime}} \backslash B_{o}\right)$ aus einer exakten Homologiefolge. Somit bleibt nur noch $\mathrm{zu}$ zeigen, daß $\Gamma$ und $\mathrm{ClDiv}^{\mathbb{T}}\left(X_{\Delta^{\prime}}\right)$ abstrakt isomorphe Gruppen sind. Nun gilt $\Gamma \cong$ $\operatorname{coker}\left[\varphi: \mathbb{Z}^{n} \rightarrow N\right]$ mit $\varphi\left(e_{i}\right)=v_{i}$, wobei $e_{1}, \ldots, e_{n}$ die kanonische Basis von $\mathbb{Z}^{n}$ ist, und $\mathrm{ClDiv}^{\mathbb{T}}\left(X_{\Delta^{\prime}}\right) \cong \operatorname{coker}\left[\psi: M \rightarrow \mathbb{Z}^{n}\right]$ mit $\psi(\chi)=\left(\left\langle\chi, v_{i}\right\rangle\right)_{i=1, \ldots, n}$ für $\chi \in M$. Da $\varphi$ und $\psi$ zueinander transponierte injektive Homomorphismen von Gittern mit gleichem Rang $n$ sind, haben sie die gleichen invarianten Faktoren und somit isomorphe Kokerne, was zu zeigen war.

Für $X:=X_{\Delta}$ erhalten wir die Homologieaussagen des Satzes durch Vergleich der zu den offenen Inklusionen $X_{\Delta^{\prime}} \stackrel{\varsigma}{\rightarrow} X_{\Delta^{(1)}} \stackrel{\varsigma}{\rightarrow} X_{\Delta}$ auf dem Niveau der Gruppen $H_{2 n-j}^{\text {cld }}$ bzw. ClDiv ${ }_{W}^{\mathbb{T}}$ gehörigen Beschränkungsabbildungen. Die Aussagen zur Kohomologie lassen sich nun mit Hilfe der singulären Poincaré-Dualitätstheorie (siehe $\left[\mathrm{Kp}_{1,2}\right]$ ) herleiten. Dazu wird die lokale Homologie $\mathcal{H}_{2 n-j}$ für $j=1,2$ benötigt. Indem man die Homologieaussagen auf affine torische Varietäten $X_{\sigma}$ anwendet (und im entarteten Fall, d.h. für $d(\sigma)<n$, zusätzlich die Künnethformel benutzt), erhält man $\mathcal{H}_{2 n-1}=0$ sowie $H^{0}\left(X_{\sigma}, \mathcal{H}_{2 n-2}\right)=$ $\mathrm{ClDiv}{ }_{\mathrm{W}}^{\mathbb{T}}\left(X_{\sigma}\right)$. Wegen $\mathcal{H}_{2 n-1}=0$ gibt es nun eine exakte Folge

$$
0 \longrightarrow H^{2}(X) \stackrel{P_{2 n-2}}{\longrightarrow} H_{2 n-2}^{\text {cld }}(X) \longrightarrow H^{0}\left(X, \mathcal{H}_{2 n-2}\right)
$$

und somit den ersten Isomorphismus in der Kette

$$
\begin{aligned}
& H^{2}(X) \cong \operatorname{im} P_{2 n-2}=\operatorname{ker}\left[H_{2 n-2}^{\mathrm{cld}}(X)\right.\left.\rightarrow H^{0}\left(X, \mathcal{H}_{2 n-2}\right)\right] \cong \\
& \operatorname{ker}\left[\vartheta: \operatorname{ClDiv}_{\mathrm{W}}^{\mathbb{T}}(X) \longrightarrow \bigoplus_{\sigma} \operatorname{ClDiv}_{\mathrm{W}}^{\mathbb{T}}\left(X_{\sigma}\right)\right] .
\end{aligned}
$$

Der zweite Isomorphismus folgt aus der kanonischen Inklusion von $H^{0}\left(X, \mathcal{H}_{2 n-2}\right)$ in die Gruppe $\bigoplus_{\sigma} H^{0}\left(X_{\sigma}, \mathcal{H}_{2 n-2}\right)$, die schließlich mit $\bigoplus_{\sigma} \mathrm{ClDiv}_{\mathrm{W}}^{\mathbb{T}}\left(X_{\sigma}\right)$ identifiziert wird. Es ist klar, daß ker $\vartheta$ in der Divisorenklassengruppe $\mathrm{ClDiv}_{\mathrm{C}}^{\mathbb{T}}(X)$ enthalten ist; die umgekehrte Inklusion folgt daraus, daß ein invarianter Cartier-Divisor auf einer affinen torischen Varietät bereits ein Hauptdivisor ist.

Bei dem hier skizzierten Beweis folgen die Kohomologieaussagen von Satz 1 aus denen zur Homologie. Umgekehrt lassen sich mit Hilfe der Poincaré-Dualität für die glatte offene invariante Teilmenge $V_{1}:=X_{\Delta(\leq 1)}$ die Homologie- aus den Kohomologieaussagen folgern, weil jeder Weil-Divisor auf $V_{1}$ schon ein Cartier-Divisor ist und die offene Inklusion $V_{1} \subset$ $X_{\Delta}$ Isomorphismen für $\mathrm{ClDiv} \mathrm{W}_{\mathrm{W}}^{\mathbb{T}}$ und für $H_{2 n-j}^{\text {cld }}$ mit $j \leqq 2$ induziert.

Unser in $[\mathrm{BF}]$ ausgeführter früherer Beweis gilt nur im kompakten Fall und benutzt die Auflösung der Singularitäten sowie die „divisorielle“ Teilaussage des Satzes von Jurkiewicz-Danilov, die sich jetzt als Spezialfall ergibt. 
3. Beweis von Satz 2. Aufgrund der Kohomologieaussagen von Satz 1 folgt die Behauptung aus der - auch im entarteten Fall gültigen - Identifikation von $I_{\mathfrak{p}} H_{2 n-j}^{\text {cld }}(X)$ mit $H^{j}\left(V_{\mathfrak{p}}\right)$ für $j \leqq 2$, die wir aus der garbentheoretischen Interpretation $I_{\mathfrak{p}} H_{2 n-j}^{\text {cld }}(X)=$ $\mathbb{H}^{j}\left(X, \mathcal{P}_{\mathfrak{p}}\right)$ der Schnitthomologie als Hyperkohomologie des „Deligne-Komplexes“ $\mathcal{P}_{\mathfrak{p}}$ wie folgt erhalten: Wir betrachten die invarianten offenen Teilmengen

$$
V_{i}=V_{i}(X):=\bigcup_{\operatorname{dim} \sigma \leqq i} B_{\sigma}
$$

für $i=0, \ldots, n$, also die Vereinigung der Torusbahnen $B_{\sigma}$ mit $\operatorname{dim} B_{\sigma} \geqq n-i$. Offenbar ist $V_{i}$ die durch den Teilfächer $\Delta^{(\leqq i)}$ definierte torische Varietät. Für $i=0$ erhalten wir den eingebetteten Torus $V_{0}=B_{o} \cong \mathbb{T}$; die Menge $V_{1}$ ist glatt, und $V_{2}$ wird durch einen simplizialen Fächer definiert; weiter gilt $V_{i}=X$ für $i \geqq \max _{\sigma \in \Delta} \operatorname{dim} \sigma$ sowie definitionsgemäß $V_{\mathfrak{p}}:=V_{i(\mathfrak{p})}$.

Die Folge $\left(X_{j}\right)_{-1 \leqq j \leqq n}$ der dazu komplementären invarianten abgeschlossenen Untervarietäten $X_{j}:=X \backslash V_{n-j-1} \operatorname{mit}_{\operatorname{dim}_{\mathbb{C}}} X_{j}=j$ ist eine aufsteigende Filtrierung von $X$. Aufgrund der natürlichen äquivarianten lokalen Produktstruktur $\left(X_{\sigma}, B_{\sigma}\right) \cong$ $\left(Y_{\sigma},\left\{y_{\sigma}\right\}\right) \times\left(\mathbb{C}^{*}\right)^{n-d}$ längs jeder Bahn $B_{\sigma}$ wird die torische Varietät $X$ durch diese Filtrierung mit der Struktur einer stratifizierten Pseudomannigfaltigkeit im Sinne von [Bo: $\mathrm{V}, \S 1]$ versehen.

Wir wollen nun einen natürlichen Isomorphismus

$$
I_{\mathfrak{p}} H_{2 n-2}^{\text {cld }}(X) \cong H^{2}\left(V_{\mathfrak{p}}\right)
$$

etablieren, womit dann Satz 2 im nicht entarteten Fall aus Satz 1 folgt, da mit $X$ auch $V_{\mathfrak{p}}$ nicht entartet ist. Dazu erinnern wir an die Beschreibung der Schnitthomologie $I_{\mathfrak{p}} H_{2 n-q}^{\text {cld }}(X)$ mit abgeschlossenen Trägern als Hyperkohomologie $\mathbb{H}^{q}\left(X, \mathcal{P}_{\mathfrak{p}}\right)$ des ,DeligneKomplexes" $\mathcal{P}_{\mathfrak{p}}^{\bullet}$ in einer geeigneten derivierten Kategorie (vgl. [Bo: V, § 2]): Man erhält $\mathcal{P}_{\mathfrak{p}}$ durch sukzessive Fortsetzung, ausgehend von der glatten offenen Teilmenge $V_{1}$, wo der Komplex $\left.\mathcal{P}_{\mathfrak{p}}\right|_{V_{1}}$ durch die konstante Garbe $\mathbb{Z}_{V_{1}}$ gegeben ist. Bezeichnet $j: V_{i-1} \stackrel{\sigma}{\rightarrow} V_{i}$ für $i \geqq 2$ die natürliche offene Inklusion, so setzt man

$$
\left.\mathcal{P}_{\mathfrak{p}}^{\bullet}\right|_{V_{i}}:=\tau_{\leqq \mathfrak{p}(2 i)} \mathbf{R} j_{*}\left(\left.\mathcal{P}_{\mathfrak{p}}^{\bullet}\right|_{V_{i-1}}\right) \text {. }
$$

Aus den Eigenschaften des Funktors $\tau$ ist leicht zu sehen, daß für jede Toleranzfunktion $\mathfrak{p}$ die Hyperkohomologie-Garbe $\mathcal{H}^{0} \mathcal{P}_{\mathfrak{p}}$ die konstante Garbe $\mathbb{Z}_{X}$ ist und daß $\mathcal{H}^{1} \mathcal{P}_{\mathfrak{p}}$ stets eine Untergarbe von $\mathcal{H}^{1} \mathcal{P}_{\mathfrak{t}}$ ist (für ein allgemeineres Ergebnis sei auf [Kp, Fi: 3.5$]$ verwiesen). Mit dem Isomorphismus $\mathcal{H}^{j} \mathcal{P}_{\mathfrak{t}} \cong \mathcal{H}_{2 n-j}$ (vgl. [Bo: V, 2.12]) folgt daher aus $\mathcal{H}_{2 n-1}=0$ sofort $\mathcal{H}^{1} \mathcal{P}_{\mathfrak{p}}^{\bullet}=0$ und damit $I_{\mathfrak{p}} H_{2 n-1}^{\text {cld }}(X)=0$. Weiter erhalten wir einen Quasi-Isomorphismus

$$
\left.\mathcal{P}_{\mathfrak{p}}\right|_{V_{i}} \cong \mathbb{Z}_{V_{i}} \text { für } \mathfrak{p}(2 i) \leqq 1
$$

Andererseits folgt aus (3.2) ein Quasi-Isomorphismus

$$
\tau_{\leqq q} \mathcal{P}_{\mathfrak{p}} \cong \tau_{\leqq q} \mathbf{R} j_{*}\left(\left.\mathcal{P}_{\mathfrak{p}}\right|_{V_{i}}\right) \quad \text { für } \quad q \leqq \mathfrak{p}(2 i+2) \text { oder } i=n
$$

wobei jetzt $j$ die natürliche offene Inklusionsabbildung $V_{i} \stackrel{\varsigma}{\rightarrow} X$ bezeichnet. Daraus erhalten wir einen Isomorphismus

$$
\mathbb{H}^{q}\left(X, \mathcal{P}_{\mathfrak{p}}^{\bullet}\right) \cong \mathbb{H}^{q}\left(V_{i}, \mathcal{P}_{\mathfrak{p}}^{\bullet}\right) \quad \text { für } \quad q \leqq \mathfrak{p}(2 i+2) \text { oder } i=n
$$

Für $i=i(\mathfrak{p})$ gilt nun gleichzeitig $\mathfrak{p}(2 i) \leqq 1$ sowie $\mathfrak{p}(2 i+2) \geqq 2$ oder $i=n$. Mit den Formeln (3.3) und (3.4) für $q=2$ und $i=i(\mathfrak{p})$ erhalten wir den gesuchten Isomorphismus 
(3.1) aus der Kette

$$
H^{2}\left(V_{\mathfrak{p}}\right)=\mathbb{H}^{2}\left(V_{\mathfrak{p}}, \mathbb{Z}_{V_{\mathfrak{p}}}\right) \cong \mathbb{H}^{2}\left(V_{\mathfrak{p}}, \mathcal{P}_{\mathfrak{p}}^{\bullet}\right)=\mathbb{H}^{2}\left(X, \mathcal{P}_{\mathfrak{p}}^{\bullet}\right)=I_{\mathfrak{p}} H_{2 n-2}^{\text {cld }}(X)
$$

Damit ist der Beweis für den nicht entarteten Fall abgeschlossen. Im entarteten Fall induziert die Produktzerlegung $X \cong Y \times \mathbb{T}^{\prime \prime}$ für jedes $i \geqq 0$ eine analoge Zerlegung $V_{i}(X) \cong V_{i}(Y) \times \mathbb{T}^{\prime \prime}$ und somit auch $V_{\mathfrak{p}}(X) \cong V_{\mathfrak{p}}(Y) \times \mathbb{T}^{\prime \prime}$. Der Isomorphismus $I_{\mathfrak{p}} H_{2 n-2}^{\text {cld }}(X) \cong \mathrm{ClDiv}_{\mathfrak{p}}^{\mathbb{T}}(X) \oplus H^{2}\left(\mathbb{T}^{\prime \prime}\right)$ des Zusatzes - wobei wieder $\mathbb{T}^{\prime \prime}$ maximal, also $Y$ nicht entartet, ist - ergibt sich nun leicht durch Kombination des Isomorphismus aus (3.1), der Künnethformel $H^{2}\left(V_{\mathfrak{p}}(X)\right) \cong H^{2}\left(V_{\mathfrak{p}}(Y)\right) \oplus H^{2}\left(\mathbb{T}^{\prime \prime}\right)$, Satz 1 für $V_{\mathfrak{p}}(Y)$ sowie dem Isomorphismus $\operatorname{ClDiv}_{\mathfrak{p}}^{\mathbb{T}}(X) \cong \operatorname{ClDiv}_{\mathfrak{p}}^{\mathbb{T}}(Y)$.

4. Die Schnitthomologie-Bettizahl $I_{\mathfrak{p}} b_{2 n-2}^{\text {cld }}(X)$. Wir betrachten jetzt wieder nicht entartete torische Varietäten (im entarteten Fall ist zu den betrachteten (Schnitt-)Bettizahlen noch $b_{2}\left(\mathbb{T}^{\prime \prime}\right)$ zu addieren). Für die Bettizahlen der invarianten offenen Teilmengen $V_{i}$ von $X$ (siehe $\left.\S 3\right)$ mit $1 \leqq i \leqq n$ liefert Satz 1 mit Hilfe der Rangaussagen am Ende von $\S 1$ die Formeln

$$
b_{2 n-2}^{\text {cld }}\left(V_{i}\right)=\operatorname{card} \Delta^{(1)}-n \quad \text { und } \quad b_{2}\left(V_{i}\right)=b_{2 n-2}^{\text {cld }}\left(V_{i}\right)-\operatorname{rg} R\left(\Delta^{(\leqq i)}\right),
$$

wobei $R\left(\Delta^{(\leqq i)}\right)$ eine Relationenmatrix für den Teilfächer $\Delta^{(\leqq i)}$ ist. (Eine solche Matrix ist nicht eindeutig bestimmt, wohl aber ihr Rang.) Mit dem Beweis von Satz 2 erhalten wir daraus für jede Toleranzfunktion $\mathfrak{p}$ die Schnitthomologie-Bettizahl

$$
I_{\mathfrak{p}} b_{2 n-2}^{\text {cld }}\left(X_{\Delta}\right):=\operatorname{rg} I_{\mathfrak{p}} H_{2 n-2}^{\text {cld }}\left(X_{\Delta}\right)=b_{2}\left(V_{\mathfrak{p}}\right)=\operatorname{card} \Delta^{(1)}-n-\operatorname{rg} R\left(\Delta^{(\mathfrak{p})}\right),
$$

wobei wir $\Delta^{(\mathfrak{p})}:=\Delta^{(\leqq i(\mathfrak{p}))}$ setzen.

Nach Konstruktion von $R$ sind für jeden Kegel $\sigma \in \Delta$ die Zeilen $\left(r_{v w}^{\sigma}\right)_{w \in P(\Delta)}$ für $v \in P(\sigma) \backslash P^{\prime}(\sigma)$ linear unabhängig, und damit ergibt sich der Rang der zugehörigen Teilmatrix $R_{\sigma}$ sofort zu $\operatorname{rg}\left(R_{\sigma}\right)=\operatorname{card} P(\sigma)-\operatorname{dim}(\sigma)$. Es ist jetzt leicht, eine vierdimensionale affine torischen Varietät $X_{\sigma}$ zu finden, deren Schnitthomologie-Bettizahl $I_{\mathfrak{p}} b_{6}^{\text {cld }}\left(X_{\sigma}\right)=b_{2}\left(V_{\mathfrak{p}}\right)$ je nach Wahl von $\mathfrak{p}$ für $i(\mathfrak{p})=4,3,2$ die Werte 0 , 1 oder 2 annimmt. Dazu muß offenbar $b_{2}\left(V_{4-j}\right)=j$ für $j=0,1,2$ gelten, und das trifft zu, falls $\sigma=\operatorname{pos} \operatorname{lin}\left(v_{1}, \ldots, v_{6}\right)$ ein vierdimensionaler Kegel mit sechs Kanten in $\mathbb{R}^{4}$ ist, der genau eine dreidimensionale Seite $\tau=\operatorname{pos} \operatorname{lin}\left(v_{1}, \ldots, v_{4}\right)$ mit vier Kanten und sonst nur simpliziale Seiten enthält. Für ein explizites Beispiel wähle man etwa $v_{i}$ als Spaltenvektoren der Matrix

$$
\left(\begin{array}{rrrrrr}
1 & 0 & -1 & 0 & 1 & -1 \\
0 & 1 & 0 & -1 & 0 & 0 \\
-1 & -1 & -1 & -1 & 1 & 1 \\
1 & 1 & 1 & 1 & 1 & 1
\end{array}\right)
$$

Diese affine Varietät $X_{\sigma}$ kann auf minimale Weise zu einer kompakten (sogar projektiven) torischen Varietät $X=X_{\Delta}$ vervollständigt werden: Für einen primitiven Gittervektor $v_{0}$ im Inneren des Kegels $\sigma$ betrachtet man mit $v_{7}:=-v_{0}$ und $\rho_{7}:=\mathbb{R}_{\geqq 0} v_{7}$ den vollständigen Fächer $\Delta$ mit $\Delta^{\max }=\{\sigma\} \cup\left\{\tau+\rho_{7} ; \tau \in \sigma^{(3)}\right\}$; d.h. man nimmt zu $\sigma$ und seinen Seiten alle Kegel und deren Seiten hinzu, die von den Seiten von $\sigma$ und dem Strahl $\rho_{7}$ erzeugt werden. Da der Kegel $\sigma$ genau eine nicht-simpliziale Seite hat, nämlich pos lin $\left(v_{1}, \ldots, v_{4}\right)$, ergibt sich genau ein neuer nicht-simplizialer vierdimensionalen Kegel mit fünf Kanten, aber ohne „neue“ Relationen; alle anderen „neuen“ Kegel 
sind simplizial. Für diese Varietät gilt $b_{4}(X)=b_{2}\left(V_{2}\right)=7-4=3, b_{2}(X)=b_{4}-2=1$ und $b_{2}\left(V_{3}\right)=b_{4}-1=2$.

Aus der Formel (4.1) folgt, daß $b_{2 n-2}^{\text {cld }}\left(V_{i}\right)=b_{2 n-2}^{\text {cld }}(X)$ immer eine kombinatorische Invariante des definierenden Fächers $\Delta$ ist ( $\operatorname{vgl}$. [BBFK]). Weiter gilt $\operatorname{rg} R\left(\Delta^{(\leqq i)}\right)=0$ und damit $b_{2}\left(V_{i}\right)=b_{2 n-2}^{\text {cld }}\left(V_{i}\right)$ genau dann, wenn $\Delta^{(\leqq i)}$ simplizial ist. Insbesondere trifft das auf $i=2 \mathrm{zu}$, und damit hängt wegen $i(\mathfrak{m})=2$ die Schnitthomologie-Bettizahl $I b_{2 n-2}^{\text {cld }}\left(X_{\Delta}\right):=I_{\mathfrak{m}} b_{2 n-2}^{\text {cld }}\left(X_{\Delta}\right)=b_{2}\left(V_{2}\right)$ nur vom kombinatorischen Typ des Fächers ab.

Sobald ein Fächer mehrere nicht-simpliziale Kegel hat, sind die Bettizahlen $b_{2}\left(V_{i}\right)$ für $i \geqq 3$ und insbesondere die Schnitthomologie-Bettizahlen $I_{\mathfrak{p}} b_{2 n-2}^{\text {cld }}(X)=b_{2}\left(V_{\mathfrak{p}}\right)$ für $\mathfrak{p}<\mathfrak{m}$ im allgemeinen keine kombinatorischen Invarianten des Fächers $\Delta$, da die Ränge der Relationenmatrizen $R\left(\Delta^{(\leqq i)}\right)$ nicht kombinatorisch invariant sind. Nun ist für zwei Kegel $\sigma, \sigma^{\prime} \in \Delta$ jede Zeile von $R_{\sigma^{\prime}}$, die zu einem Vektor $v^{\prime} \in P\left(\sigma^{\prime}\right) \backslash P^{\prime}\left(\sigma^{\prime}\right)$ mit $v^{\prime} \notin P(\sigma)$ gehört, aus den Zeilen von $R_{\sigma}$ nicht linear kombinierbar und vergrößert somit den Rang. Eine solche Abschätzung zeigt, daß der dreidimensionale Fächer $\Delta\left(\sigma_{1}, \sigma_{2}, \sigma_{3}\right)$ mit $\sigma_{1}=\operatorname{pos} \operatorname{lin}\left\{v_{1}, v_{2}, v_{4}, v_{5}\right\}, \sigma_{2}=\operatorname{pos}\left\{v_{2}, v_{3}, v_{5}, v_{6}\right\}$ und $\sigma_{3}=\operatorname{pos} \operatorname{lin}\left\{v_{3}, v_{1}, v_{6}, v_{4}\right\}$, der von den drei vierkantigen dreidimensionalen Kegeln $\sigma_{i}$ erzeugt wird, die zu den rechteckigen Seitenflächen eines Dreikantprismas gehören, den kleinsten Typ repräsentiert, für den $\operatorname{rg} R(\Delta)$ und somit auch $b_{2}\left(X_{\Delta}\right)$ keine kombinatorische Invariante ist. Ein Beispiel liefern $v_{1}=(1,1,1), v_{2}=(-1,1,1), v_{3}=(0,-1,1)$ und $v_{i}=v_{i-3}-2 e_{3}$ für $i=4,5,6$ sowie $v_{1}^{\prime}=2 v_{1}-v_{2}=(3,1,1)$ und $v_{i}^{\prime}=v_{i}$ für $i=2, \ldots, 6$ : es gilt $\operatorname{rg} R(\Delta)=2$ und $\operatorname{rg} R\left(\Delta^{\prime}\right)=3$ und damit $b_{2}\left(X_{\Delta}\right)=1$ und $b_{2}\left(X_{\Delta^{\prime}}\right)=0$. Diese Fächer lassen sich durch die beiden simplizialen Kegel $\delta_{1}:=\operatorname{pos} \operatorname{lin}\left\{v_{1}, v_{2}, v_{3}\right\}$ bzw. $\delta_{1}^{\prime}:=\operatorname{pos} \operatorname{lin}\left\{v_{1}^{\prime}, v_{2}, v_{3}\right\}$ sowie $\delta_{2}=\delta_{2}^{\prime}:=\operatorname{pos} \operatorname{lin}\left\{v_{4}, v_{5}, v_{6}\right\}$ vervollständigen; für die zugehörigen kompakten dreidimensionalen torischen Varietäten gilt ebenfalls $b_{2}=1$ bzw. $b_{2}=0$. - Dieses Beispiel geht auf M. Eikelberg zurück, der solche Fragen in $\left[\mathrm{Ei}_{1,2}\right]$ systematisch untersucht hat.

In Dimension $n \geqq 4$ kann man entsprechend zeigen, daß auch die SchnitthomologieBettizahlen $I_{\mathfrak{p}} b_{2 n-2}=b_{2}\left(V_{\mathfrak{p}}\right)$ mit $2<i(\mathfrak{p})<n$ keine kombinatorischen Invarianten sind. Vierdimensionale affine Beispiele ergeben sich ähnlich wie oben, indem man von dreidimensionalen projektiven Varietäten ausgeht, für die $b_{2}$ keine kombinatorische Invariante ist. Solche dreidimensionale Fächer wurden zuerst von M. McConnell angegeben; wir folgen einem „kleineren“ Beispiel von M. Eikelberg: Für die Kegel $\sigma=\operatorname{pos} \operatorname{lin}\left(v_{1}, \ldots, v_{8}\right)$ bzw. $\sigma^{\prime}=\operatorname{pos} \operatorname{lin}\left(v_{1}^{\prime}, \ldots, v_{8}^{\prime}\right)$, die von den Spalten der Matrizen

$$
\left(\begin{array}{rrrrrrrr}
0 & 0 & 0 & 0 & 2 & 2 & -2 & -1 \\
0 & 3 & 0 & -3 & 0 & 1 & 0 & 1 \\
3 & 0 & -3 & 0 & 1 & 0 & 1 & 0 \\
1 & 1 & 1 & 1 & 1 & 1 & 1 & 1
\end{array}\right),\left(\begin{array}{cccccccc}
-20 & 20 & 0 & 0 & 40 & 40 & -45 & -36 \\
0 & 40 & 0 & -60 & 0 & 20 & 0 & 12 \\
80 & 0 & -60 & 0 & 20 & 0 & 30 & 0 \\
1 & 1 & 1 & 1 & 1 & 1 & 1 & 1
\end{array}\right)
$$

erzeugt werden, gilt $\operatorname{rg} R\left(\sigma^{(\leqq 3)}\right)=3$ und $\operatorname{rg} R\left(\sigma^{\prime(\leqq 3)}\right)=4$ (vgl. [ $\left.\operatorname{Ei}_{2}: 4.4\right]$ ). Damit ergeben sich die Bettizahlen $b_{6}=4, b_{2}=0$ und $b_{2}\left(V_{3}\right)=1$ für $X_{\sigma}$ bzw. $b_{2}\left(V_{3}\right)=0$ für $X_{\sigma^{\prime}}$. Wie oben können auch diese Beispiele zu projektiven Varietäten abgeschlossen werden, indem man die von einem geeigneten primitiven Gittervektor $v_{9}=v_{9}^{\prime}$, z.B. $v_{9}=v_{9}^{\prime}=(0,0,0,-1)$, und den Seiten von $\sigma$ bzw. $\sigma^{\prime}$ aufgespannten Kegel hinzunimmt. Dabei ergeben sich keine zusätzlichen Relationen; wir erhalten die Bettizahlen $b_{6}=5$, $b_{2}=1$ und $b_{2}\left(V_{3}\right)=2$ bzw. $b_{2}\left(V_{3}\right)=1$.

Neben dem kombinatorischen Typ von Fächern betrachten wir deren linearen Typ, der wie folgt definiert ist: Zwei Fächer $\Delta$ in $N_{\mathbb{R}}$ und $\Delta^{\prime}$ in $N_{\mathbb{R}}^{\prime}$ sind vom gleichen linearen Typ, wenn es einen Vektorraum-Isomorphismus $\psi: N_{\mathbb{R}} \rightarrow N_{\mathbb{R}}^{\prime}$ mit $\psi\left(\sigma^{\prime}\right) \in \Delta^{\prime}$ für 
jeden Kegel $\sigma \in \Delta$ gibt. Trivialerweise sind dann auch die Teilfächer $\Delta^{(\leqq i)}$ und $\Delta^{\prime(\leqq i)}$ für $1 \leqq i \leqq n$ jeweils vom gleichen linearen Typ. Bei der Definition wird nicht vorausgesetzt, daß die Gitter $\psi(N)$ und $N^{\prime}$ kommensurabel (bis auf einen eventuellen irrationalen Proportionalitätsfaktor) sind.

Wie wir in [BBFK: $\S 3 . \mathrm{C}]$ gezeigt haben, kann ein solcher Automorphismus $\psi$ aber stets durch einen modifizierten Automorphismus $\psi^{\prime}$ ersetzt werden, für den diese Kommensurabilität gilt. Daraus folgt leicht für die zugehörigen torischen Varietäten $X:=X_{\Delta}$ und $X^{\prime}:=X_{\Delta^{\prime}}$, daß es einen Morphismus $X \rightarrow X^{\prime}$ gibt, der eine mit den jeweiligen Torusoperationen verträgliche verzweigte Überlagerung ist. Damit kann $X^{\prime}$ als Quotient $X / \Gamma$ nach einer endlichen Untergruppe $\Gamma$ des Torus $\mathbb{T}$ dargestellt werden. In dieser Situation gibt es einen Isomorphismus $H_{\bullet}^{\Phi}(X / \Gamma, \mathbb{Q}) \cong H_{\bullet}^{\Phi}(X, \mathbb{Q})^{\Gamma}$ in der rationalen Homologie mit Trägern in der Familie $\Phi=$ cld der abgeschlossenen sowie in der Familie $\Phi=c$ der kompakten Mengen (vgl. [Bd: II, Thm. 19.1]); da aber die Torusoperation auf der rationalen Homologie trivial ist, folgt insbesondere die Gleichheit entsprechender Bettizahlen $b_{j}^{\Phi}$ von $X$ bzw. von $X^{\prime}$.

Als Anwendung erhalten wir, daß die Schnitthomologie-Bettizahl $I_{\mathfrak{p}} b_{2 n-2}^{\text {cld }}$ für jedes $\mathfrak{p}$ eine Invariante des linearen Typs ist.

Zum Abschluß dieses Abschnittes diskutieren wir noch kurz für dreidimensionale kompakte torische Varietäten die Schnitthomologie-Bettizahlen $I b_{j}(X)=I b_{6-j}(X)$ zur mittleren Toleranz $\mathfrak{m}$. Für $j=0,1,2$ erhalten wir die Werte 1, $0, b_{4}(X)$. Nach [Fi: Thm. $1.1,1.2]$ gilt $I b_{3}(X)=0$ im projektiven Fall. Wir zeigen, daß diese Aussage auch im nicht-projektiven kompakten Fall richtig ist:

Bemerkung: Für eine dreidimensionale kompakte komplexe torische Varietät $X$ gilt $I b_{3}(X)=0$.

Beweis: Es gibt eine simpliziale Unterteilung $\hat{\Delta}$ des Fächers $\Delta$ ohne zusätzliche Strahlen (vgl. [Ew: V, 4.3]). Die zugehörige eigentliche äquivariante Modifikation $\pi$ : $\hat{X} \rightarrow X$ ist dann eine "kleine“ Abbildung, d.h. für die exzeptionelle Menge $E$ gilt $\operatorname{dim} E \leqq 1<(\operatorname{dim} X) / 2$. Nach [Go, MPh: 6.2] induziert eine solche kleine Modifikation einen Isomorphismus $I H_{\bullet}(X, \mathbb{Q}) \cong H_{\bullet}(\hat{X}, \mathbb{Q})$; insbesondere folgt $I_{\bullet}(X)=b_{\bullet}(\hat{X})$. Weil die torische Varietät $\hat{X}$ durch einen vollständigen simplizialen Fächer gegeben ist, gilt $b_{3}(\hat{X})=0$.

5. Homologie-Chernklasse $c_{n-1}$ und Schnitthomologie. Die Homologie-Chernklasse $c_{n-1}(X) \in H_{2 n-2}^{\text {cld }}(X)$ (im Sinne von M.-H. Schwartz [Sc] und R. MacPherson $[\mathrm{MPh}])$ einer torischen Varietät $X=X_{\Delta}$ wird nach einem unveröffentlichten Ergebnis von F. Ehlers (vgl. [Fu: pp. 113, 145], siehe [BBF] für einen Beweis) durch den invarianten Weil-Divisor $\sum \bar{B}_{v}$ repräsentiert, der somit im glatten Fall ein „,antikanonischer" Divisor $-K_{X}$ ist. In [BBFK: $\S 3 . \mathrm{E}$ ] haben wir die Frage diskutiert, wann diese Klasse $c_{n-1}(X)$ im Bild des Poincaré-Homomorphismus $P_{2 n-2}$ liegt. Mit Satz 2 können wir die Antwort auch auf die analoge Fragestellung für den natürlichen Vergleichshomomorphismus $\omega_{\mathfrak{p}}$ : $I_{\mathfrak{p}} H_{2 n-2}^{\text {cld }}(X) \rightarrow H_{2 n-2}^{\text {cld }}(X)$ übertragen. Wir erinnern daran, daß $\omega_{\mathfrak{p}}$ injektiv ist und wir damit $I_{\mathfrak{p}} H_{2 n-2}^{\text {cld }}(X)$ als Untergruppe von $H_{2 n-2}^{\text {cld }}(X)$ interpretieren können.

Satz 3: Für eine komplexe torische Varietät $X$ mit $\operatorname{dim} X=n$ und für eine feste Toleranzfunktion $\mathfrak{p}$ sind folgende Bedingungen äquivalent: 
(1) Die Homologie-Chernklasse $c_{n-1}(X)$ liegt im Bild des Vergleichshomomorphismus $\omega_{\mathfrak{p}}$, d.h. in der Untergruppe $\operatorname{im} \omega_{\mathfrak{p}}=I_{\mathfrak{p}} H_{2 n-2}^{\text {cld }}(X)$ von $H_{2 n-2}^{\text {cld }}(X)$;

(2) die Einschränkung des invarianten Weildivisors $\sum_{i=1}^{k(\Delta)} \bar{B}_{v}$ auf $V_{\mathfrak{p}}$ ist ein Cartierdivisor;

(3) für jeden maximalen Kegel $\sigma \in \Delta^{(\mathfrak{p})}$ gilt:

$\left(3_{\sigma}\right)$ es gibt einen Charakter $\chi_{\sigma} \in M$, so daß $\left\langle\chi_{\sigma}, v_{j}\right\rangle=1$ für alle $v_{j} \in P(\sigma)$ gilt (d.h. $P(\sigma)$ liegt in der affinen Hyperebene $\left.\left\{v \in N_{\mathbb{R}} ;\left\langle\chi_{\sigma}, v\right\rangle=1\right\}\right)$;

(4) das antikanonische Bündel $\Lambda^{n} T\left(V_{\mathfrak{p}}\right)_{\mathrm{reg}}$ des regulären Ortes von $V_{\mathfrak{p}}$ läßt sich zu einem Geradenbündel auf ganz $V_{\mathfrak{p}}$ fortsetzen;

(5) Alle Singularitäten von $V_{\mathfrak{p}}$ sind Gorenstein-Singularitäten.

Insbesondere sind (1) - (5) erfüllt, wenn gilt:

(6) Alle Singularitäten von $V_{\mathfrak{p}}$ sind vollständige Durchschnitte.

Für eine Toleranz $\mathfrak{p}$ mit $i(\mathfrak{p})=2$, also insbesondere für die mittlere Toleranz $\mathfrak{m}$, sind alle diese Bedingungen äquivalent.

Im Spezialfall $\mathfrak{p}=\mathfrak{o}$, in dem $V_{\mathfrak{o}}=X, I_{\mathfrak{o}} H_{2 n-2}^{\text {cld }}(X) \cong H^{2}(X)$ und $\operatorname{ClDiv}_{\mathfrak{o}}^{\mathbb{T}}(X)=$ $\mathrm{Cl} \operatorname{Div}_{\mathrm{C}}^{\mathbb{T}}(X)$ gilt, erhalten wir damit Bedingungen, wann eine Kohomologieklasse $c^{1}(X) \in$ $H^{2}(X)$ mit $P_{2 n-2}\left(c^{1}\right)=c_{n-1}$ existiert. Eine solche „Chern"klasse in der Kohomologie ist dann eindeutig bestimmt.

Zum B eweis des Satzes bemerken wir, daß es aufgrund der natürlichen Identifikation $I_{\mathfrak{p}} H_{2 n-2}^{\text {cld }}(X) \cong H^{2}\left(V_{\mathfrak{p}}\right)$ genügt, den Fall $\mathfrak{p}=\mathfrak{o}$, also $V_{\mathfrak{p}}=X$, zu betrachten. Die Äquivalenz der Bedingungen (1) - (3) und die Implikation (2) $\Longrightarrow$ (4) sind unmittelbar zu sehen. Zum Nachweis der Implikation $(4) \Longrightarrow(1)$, die übrigens für jede normale algebraische Varietät gilt, sei $L$ eine Fortsetzung des antikanonischen Bündels $\bigwedge^{n} T\left(X_{\text {reg }}\right)$ zu einem Geradenbündel auf $V_{\mathfrak{p}}$ gemäß (4). Für eine Auflösung $\pi: \hat{X} \rightarrow X$ der Singularitäten gilt dann $c_{n-1}(X)=\pi_{*}\left(c_{n-1}(\hat{X})\right)$, weiter stimmen $\pi^{*}(L)$ und $\bigwedge^{n} T(\hat{X})$ außerhalb der exzeptionellen Menge von $\pi$ überein, und daraus folgt $P_{2 n-2}\left(c^{1}(L)\right)=c_{n-1}(X)$. Die Äquivalenz mit der Gorenstein-Bedingungung (5) (d.h. die Koordinatenringe sind Gorenstein-Ringe) gilt im wesentlichen nach Definition; in dieser Formulierung findet sich der Satz bei M.-N. Ishida (vgl. [Is]; für Gorenstein-Ringe verweisen wir auf [Br, He]).

Für die Implikation $(6) \Longrightarrow(4)$ benutzen wir die Adjunktionsformel $K_{Z} \cong\left(K_{Y} \otimes\right.$ $\left.\left[Z_{1}\right] \otimes \ldots \otimes\left[Z_{k}\right]\right)\left.\right|_{Z}$ für das kanonische Geradenbündel $K_{Z}$ eines glatten analytischen vollständigen Durchschnittes $Z=Z_{1} \cap \ldots \cap Z_{k}$ in einer glatten komplexen Mannigfaltigkeit $Y$ (vgl. [Di: Ch. 5, (3.8)] sowie [Gr, Ha: 1.1, p. 147]). Somit ist $K_{Z}$ die Einschränkung eines Geradenbündels auf der umgebenden Mannigfaltigkeit $Y$. Dieses existiert auch dann, wenn der vollständige Durchschnitt $Z$ nicht glatt ist. Durch Dualisieren folgt, daß eine Varietät, die nur Singularitäten vollständiger Durchschnitte hat, der Fortsetzungsbedingung (4) genügt.

Für einen simplizialen Kegel $\sigma$ können wir $\left(3_{\sigma}\right)$ durch eine äquivalente Bedingung ersetzen. Dazu benutzen wir, daß es eine bis auf Isomorphie eindeutig bestimmte endliche kleine Untergruppe $\Gamma_{\sigma} \subset \mathrm{GL}_{d}(\mathbb{C})$ (für $d:=\operatorname{dim} \sigma$ ) von Diagonalmatrizen mit $X_{\sigma} \cong$ $\mathbb{C}^{d} / \Gamma_{\sigma} \times\left(\mathbb{C}^{*}\right)^{n-d}$ existiert (vgl. $\left.\S 0\right)$.

ZusATZ Zu SATZ 3: Für einen simplizialen Kegel $\sigma$ ist die Bedingung $\left(3_{\sigma}\right)$ äquivalent $z u$

$\left(6_{\sigma}\right)$ Die Gruppe $\Gamma_{\sigma}$ ist eine Untergruppe von $\mathrm{SL}_{d}(\mathbb{C})$. 
Der Beweis findet sich in der Arbeit [Wa] von K. Watanabe.

Falls der Fächer $\Delta^{(\mathfrak{p})}$ simplizial ist - was insbesondere für die mittlere Toleranz zutrifft -, sind also (1) - (5) dazu äquivalent, daß für jeden maximalen Kegel die Bedingung $\Gamma_{\sigma} \subset \mathrm{SL}_{d}$ des obigen Zusatzes erfüllt ist, und dazu ist für zweidimensionale Kegel äquivalent, daß $X_{\sigma}$ eine Hyperflächen-Singularität ist.

Zum Abschluß sei noch angemerkt, daß die Untergruppe $I_{\mathfrak{p}} H_{2 n-2}^{\text {cld }}(X)$ genau dann endlichen Index in $H_{2 n-2}^{\text {cld }}(X)$ hat, wenn der Fächer $\Delta^{(\mathfrak{p})}$ simplizial ist. In diesem Fall liegt ein ganzzahliges Vielfaches $m c_{n-1}(X)$ mit geeignetem $m \geqq 1$ in $I_{\mathfrak{p}} H_{2 n-2}^{\text {cld }}(X)$; mit rationalen Koeffizienten gilt also $c_{n-1}(X) \in I_{\mathfrak{p}} H_{2 n-2}^{\text {cld }}(X, \mathbb{Q})$. Insbesondere trifft das wegen $i(\mathfrak{m})=2$ auf die mittlere Toleranz $\mathfrak{p}=\mathfrak{m}$ zu, da der Fächer $\Delta^{(\leqq 2)}$ immer simplizial ist. Dies ist ein Spezialfall eines allgemeinen Resultates: Für eine beliebige algebraische Varietät $X$ gilt, daß jede durch algebraische Zykel repräsentierte rationale Homologieklasse mit abgeschlossenem Träger, also insbesondere jede Homologie-Chernklasse $c_{k}(X)$, im Bild des Vergleichshomomorphismus $I H_{\bullet}^{\text {cld }}(X, \mathbb{Q}) \stackrel{\omega}{\rightarrow} H_{\bullet}^{\text {cld }}(X, \mathbb{Q})$ liegt. Für einen Beweis sei auf $[\mathrm{BBFGK}]$ verwiesen.

\section{Literatur}

[BBF] G. Barthel, J.-P. Brasselet und K.-H. Fieseler, Classes de Chern des variétés toriques singulières, C. R. Acad. Sci. Paris Sér. I Math. 315 (1992), 187-192.

[BBFGK] G. Barthel, J.-P. Brasselet, K.-H. Fieseler, O. Gabber und L. Kaup, Sur le relèvement des cycles algébriques en homologie d'intersection, Ann. of Math. (2) 141 (1995), 147-179.

[BBFK] G. Barthel, J.-P. Brasselet, K.-H. Fieseler und L. Kaup, Diviseurs invariants et homologie de variétés toriques, erscheint in Tôhoku Math. J. (2), vorauss. 1996.

[BF] G. Barthel und K.-H. Fieseler, Invariant Divisors and Homology of Compact Complex Toric Varieties, erscheint in: Proceedings of the International Geometrical Colloquium, Moscow 1993.

[Bo] A. Borel et al., Intersection Cohomology, Progr. Math. 50, Birkhäuser, Boston etc., 1984 .

[Bd] G. Bredon, Sheaf Theory, MacGraw-Hill, New York etc., 1967.

[Br, He] W. Bruns und J. Herzog, Cohen-Macaulay rings, Cambridge Univ. Press, Cambridge, 1993.

[Da] V. I. Danilov, The Geometry of Toric Varieties, Russian Math. Surveys 33:2 (1978), 97-154, engl. Übers. von Geometrija toričeskich mnogoobrazij, Uspekhi Mat. Nauk 33:2, 200 (1978), 85-134, 247.

[Di] A. Dimca, Singularities and Topology of Hypersurfaces, Springer Universitext, Springer-Verlag, New York etc., 1992.

[Eh] F. Ehlers, Eine Klasse komplexer Mannigfaltigkeiten und die Auflösung einiger isolierter Singularitäten, Math. Ann. 218 (1975), 127-156.

[Ei $\left.{ }_{1}\right] \quad$ M. Eikelberg, The Picard Group of a Compact Toric Variety, Results Math. 22 (1992), 509-527.

[Ei 2 M. Eikelberg, Picard Groups of Compact Toric Varieties and Combinatorial Classes of Fans, Results Math. 23 (1993), 251-293.

[Ew] G. Ewald, Combinatorial Convexity and Algebraic Geometry, to appear in Grad. Texts in Math., Springer-Verlag New York etc.

[Fi] K.-H. Fieseler, Rational Intersection Cohomology of Projective Toric Varieties, J. Reine Angew. Math. 413 (1991), 88-98. 
[Fi, Kp] K.-H. Fieseler und L. Kaup, On the Hard Lefschetz Theorem in Intersection Homology of Complex Varieties with Isolated Singularities, Aequationes Math. 34 (1987), 240-263.

[Fu] W. Fulton, Introduction to Toric Varieties, Ann. of Math. Stud., Princeton Univ. Press 1993.

[Go, MPh] M. Goresky and R. MacPherson, Intersection Homology II, Invent. Math. 72 (1983), 77-130.

[Gr, Ha] Ph. Griffiths and J. Harris, Principles of Algebraic Geometry, Pure Appl. Math., Wiley, New York etc., 1978.

[Is] M.-N. Ishida, Torus embeddings and dualizing complexes, Tôhoku Math. J. (2) 32 (1980), 111-146.

[Kp 1$] \quad$ L. Kaup, Poincaré-Dualität für Räume mit Normalisierung, Ann. Scuola Norm. Sup. Pisa Cl. Sci. (3) 26 (1972), 1-31.

[Kp 2$] \quad$ L. Kaup, Zur Homologie projektiv-algebraischer Varietäten, Ann. Scuola Norm. Sup. Pisa Cl. Sci. (3) 26 (1972), 479-513.

[Kp, Fi] L. Kaup und K.-H. Fieseler, Singular Poincaré Duality and Intersection Homology, in: Proceedings of the 1984 Vancouver conference in algebraic geometry, CMS Conf. Proc. 6 (1986), 113-161.

[MCo] M. McConnell, The Rational Homology of Toric Varieties is not a Combinatorial Invariant, Proc. Amer. Math. Soc. 105 (1989), 986-991.

[MPh] R. MacPherson, Chern Classes for Singular Algebraic Varieties, Ann. of Math. (2) 100 (1974), 423-432.

[Od] T. Oda, Convex Bodies and Algebraic Geometry, Ergeb. Math. Grenzgeb. (3), Bd. 15, Springer-Verlag, Berlin etc., 1988.

[Sc] M. H. Schwartz, Classes caractéristiques définies par une stratification d'une variété analytique complexe, C. R. Acad. Sci. Paris Sér. I Math. 260 (1965), 32623264 et $3535-3537$.

[Wa] K. Watanabe, Certain invariant subrings are Gorenstein I, II, Osaka J. Math. 11 (1974), 1-8, 379-388. 